Volume 133, Number 10, Pages 3109-3113

S 0002-9939(05)07979-7

Article electronically published on April 8, 2005

\title{
ON ISOMORPHISMS OF ALGEBRAS OF SMOOTH FUNCTIONS
}

\author{
JANEZ MRČUN
}

(Communicated by Jonathan M. Borwein)

\begin{abstract}
We show that for any smooth Hausdorff manifolds $M$ and $N$, which are not necessarily second-countable, paracompact or connected, any isomorphism from the algebra of smooth (real or complex) functions on $N$ to the algebra of smooth functions on $M$ is given by composition with a unique diffeomorphism from $M$ to $N$. An analogous result holds true for isomorphisms of algebras of smooth functions with compact support.
\end{abstract}

Throughout this paper, manifolds are assumed to be smooth and Hausdorff. On the other hand, we do not assume manifolds to be second-countable, paracompact or connected. Thus manifolds may have uncountably many components, and in particular any discrete space is a manifold of dimension 0 .

Choose $\mathbb{F}$ to be either $\mathbb{R}$ or $\mathbb{C}$. For a manifold $M$ we shall denote by $C^{\infty}(M, \mathbb{F})$ the commutative algebra of $\mathbb{F}$-valued smooth functions on $M$. Its subalgebra of smooth functions with compact support on $M$ will be denoted by $C_{c}^{\infty}(M, \mathbb{F})$.

The aim of this paper is to prove the following theorem, which answers the question of Alan Weinstein, formulated at the International Euroschool on Poisson Geometry, Deformation Quantisation and Group Representations in Brussels (2003):

Theorem 1. For any Hausdorff smooth manifolds $M$ and $N$ (which are not necessarily second-countable, paracompact or connected), any isomorphism of algebras of smooth functions $T: C^{\infty}(N, \mathbb{F}) \rightarrow C^{\infty}(M, \mathbb{F})$ is given by composition with a unique diffeomorphism $\tau: M \rightarrow N$.

An analogous result, which we already proved in [4], holds true for isomorphisms of algebras of smooth functions with compact support:

Theorem 2. For any Hausdorff smooth manifolds $M$ and $N$ (which are not necessarily second-countable, paracompact or connected), any isomorphism of algebras of smooth functions with compact support $C_{c}^{\infty}(N, \mathbb{F}) \rightarrow C_{c}^{\infty}(M, \mathbb{F})$ is given by composition with a unique diffeomorphism $M \rightarrow N$.

Although we have chosen to work in the smooth context, we could as well consider manifolds of differentiability class $C^{r}$, for any $r=0,1, \ldots$ In that case we obtain an analogous result for the isomorphisms of algebras of $C^{r}$-functions (with compact support), without any changes in the proof. In particular, the result holds true

Received by the editors May 18, 2004.

2000 Mathematics Subject Classification. Primary 58A05; Secondary 46E25.

This work was supported in part by the Slovenian Ministry of Science.

(C)2005 American Mathematical Society 
for isomorphisms of algebras of continuous functions (with compact support) on Hausdorff topological manifolds.

The standard way to prove this kind of a statement is to establish a one-to-one correspondence between points of $M$ and multiplicative functionals on the algebra of smooth functions on $M$ equipped with a suitable topology. However, for this one usually needs to impose additional conditions on the spaces $M$ and $N$ (see e.g. [1, 2, 3, 5,6$]$ ).

In the proof of Theorem 1 we shall choose a different, purely algebraic approach. We shall characterize points of a manifold by suitable 'characteristic' sequences of functions on the manifold. We used these sequences already in [4], where we proved Theorem 2 In fact, the proof of Theorem 1 given below also proves Theorem 2 if in the argument one substitutes smooth functions with smooth functions with compact support.

A characteristic sequence of functions on a manifold $M$ at a point $x \in M$ is a sequence $\left(f_{n}\right)_{n}$ of $\mathbb{F}$-valued smooth functions on $M$ such that

(i) $f_{n} f_{n+1}=f_{n+1}$ for any $n=1,2, \ldots$, and

(ii) the sequence of supports $\left(\operatorname{supp}\left(f_{n}\right)\right)_{n}$ is a fundamental system of neighbourhoods of the point $x$ in $M$.

Note that the equality $f_{n} f_{n+1}=f_{n+1}$ implies that $f_{n}\left(x^{\prime}\right)=1$ for any $x^{\prime} \in$ $\operatorname{supp}\left(f_{n+1}\right)$, and hence $\operatorname{supp}\left(f_{n}\right)$ is a neighbourhood of $\operatorname{supp}\left(f_{n+1}\right)$. In particular, if $\left(f_{n}\right)_{n}$ is a characteristic sequence of functions on $M$ at $x$, then $f_{n}(x)=1$ for any $n=1,2, \ldots$. Also note that for any $x \in M$ there exists a characteristic sequence of functions at $x$.

Lemma 3. Let $\left(f_{n}\right)_{n}$ be a sequence of $\mathbb{F}$-valued smooth functions on a manifold $M$ satisfying $f_{n} f_{n+1}=f_{n+1}$ for any $n=1,2, \ldots$ Then $\left(f_{n}\right)_{n}$ is a characteristic sequence of functions at a point $x \in M$ if and only if $\bigcap_{n=1}^{\infty} \operatorname{supp}\left(f_{n}\right)=\{x\}$ and at least one of the functions in the sequence $\left(f_{n}\right)_{n}$ has compact support.

Proof. If $\left(\operatorname{supp}\left(f_{n}\right)\right)_{n}$ is a fundamental system of neighbourhoods of $x$, then there exists $m \geq 1$ such that $\operatorname{supp}\left(f_{m}\right)$ is a subset of a compact neighbourhood of $x$ and hence is itself compact. Conversely, if $\bigcap_{n=1}^{\infty} \operatorname{supp}\left(f_{n}\right)=\{x\}$ and $\operatorname{supp}\left(f_{m}\right)$ is compact for some $m \geq 1$, then first note that $\operatorname{supp}\left(f_{n}\right)$ is compact for any $n \geq m$ as well. Since for any open neighbourhood $U$ of $x$ in $M$ the intersection of the descending sequence of compact sets $\bigcap_{n=m}^{\infty}\left(\operatorname{supp}\left(f_{n}\right)-U\right)$ is empty, it follows that $\operatorname{supp}\left(f_{n}\right)-U$ is empty for some $n \geq m$; thus $\operatorname{supp}\left(f_{n}\right) \subset U$.

The idea of the proof of Theorem 1 is to show that the image of a characteristic sequence of functions along an isomorphism of algebras is again a characteristic sequence of functions, and then use this to define a map between manifolds with the required properties. The first part of this plan is done by the following lemma.

Lemma 4. Let $M$ and $N$ be manifolds and let $T: C^{\infty}(N, \mathbb{F}) \rightarrow C^{\infty}(M, \mathbb{F})$ be an isomorphism of algebras.

(i) If $\left(g_{n}\right)_{n}$ is a characteristic sequence of functions on $N$ at a point $y \in N$, then $\left(T\left(g_{n}\right)\right)_{n}$ is a characteristic sequence of functions on $M$ at a point $x \in M$.

(ii) If $\left(g_{n}\right)_{n}$ and $\left(g_{n}^{\prime}\right)_{n}$ are two characteristic sequences of functions on $N$ at the same point $y \in N$, then $\left(T\left(g_{n}\right)\right)_{n}$ and $\left(T\left(g_{n}^{\prime}\right)\right)_{n}$ are characteristic sequences of functions on $M$ at the same point $x \in M$. 
Proof. (i) For any $n=1,2, \ldots$ write $f_{n}=T\left(g_{n}\right)$. Clearly we have $f_{n} f_{n+1}=f_{n+1}$, so $f_{n}\left(x^{\prime}\right)=1$ for any $x^{\prime} \in \operatorname{supp}\left(f_{n+1}\right)$, and thus $\operatorname{supp}\left(f_{n}\right)$ is a neighbourhood of $\operatorname{supp}\left(f_{n+1}\right)$. Put

$$
K=\bigcap_{n=1}^{\infty} \operatorname{supp}\left(f_{n}\right) .
$$

First we will show that $K$ is not empty. Suppose the opposite, that $K$ is empty. Since each $f_{n}$ is non-zero, we can choose a strictly increasing sequence $\left(i_{k}\right)_{k}$ of natural numbers such that $\operatorname{supp}\left(f_{i_{k}}\right)-\operatorname{supp}\left(f_{i_{k}+1}\right)$ is non-empty for any $k=1,2, \ldots$ This implies that

$$
U_{k}=\operatorname{Int}_{M}\left(\operatorname{supp}\left(f_{i_{k}}\right)\right)-\operatorname{supp}\left(f_{i_{k}+1}\right)
$$

is a non-empty open subset of $M$. Since $K$ is assumed to be empty, the disjoint sequence of open subsets $\left(U_{k}\right)_{k}$ is locally finite in $M$. This means that we can choose a smooth function $f$ on $M$ and a sequence $\left(u_{k}\right)_{k}$ of smooth functions on $M$ such that $\operatorname{supp}\left(u_{k}\right)$ is a non-empty compact subset of $U_{k}$,

$$
f u_{2 k}=u_{2 k}
$$

and

$$
f u_{2 k+1}=0
$$

for any $k=1,2, \ldots$. Note that $f_{i_{k}} u_{k} \neq 0$. Put $g=T^{-1}(f)$ and $v_{k}=T^{-1}\left(u_{k}\right)$. Since $g_{i_{k}} v_{k}=T^{-1}\left(f_{i_{k}} u_{k}\right) \neq 0$, we can choose a point $y_{k} \in N$ such that

$$
g_{i_{k}}\left(y_{k}\right) v_{k}\left(y_{k}\right) \neq 0 \text {. }
$$

In particular we have $y_{k} \in \operatorname{supp}\left(g_{i_{k}}\right)$, so the sequence $\left(y_{k}\right)_{k}$ converges to $y$ because $\left(g_{n}\right)_{n}$ is a characteristic sequence of functions at $y$. Next, we have $g v_{2 k}=v_{2 k}$ and $g v_{2 k+1}=0$, and since $v_{2 k}\left(y_{2 k}\right) \neq 0$ and $v_{2 k+1}\left(y_{2 k+1}\right) \neq 0$, it follows that $g\left(y_{2 k}\right)=1$ and $g\left(y_{2 k+1}\right)=0$. As the sequence $\left(y_{k}\right)_{k}$ is convergent, this contradicts the continuity of $g$. This proves that $K$ is not empty.

Take a point $x \in K$, and let $U$ be an open neighbourhood of $x$ in $M$. Choose a characteristic sequence $\left(\alpha_{n}\right)_{n}$ of functions on $M$ at $x$ such that $\operatorname{supp}\left(\alpha_{1}\right) \subset U$. Put $\beta_{n}=T^{-1}\left(\alpha_{n}\right)$ and $\gamma_{n}=\beta_{n} g_{n}$. Note that

$$
\gamma_{n} \gamma_{n+1}=\gamma_{n+1} \text {. }
$$

Since $\alpha_{n}(x)=f_{n}(x)=1$, we have $\alpha_{n} f_{n} \neq 0$ and hence $\gamma_{n} \neq 0$. It follows that $\left(\operatorname{supp}\left(\gamma_{n}\right)\right)_{n}$ is a descending sequence of non-empty sets. Since $\left(g_{n}\right)_{n}$ is a characteristic sequence, there exists $m \geq 1$ such that $\operatorname{supp}\left(g_{n}\right)$ is compact for any $n \geq m$. As $\operatorname{supp}\left(\gamma_{n}\right) \subset \operatorname{supp}\left(g_{n}\right)$, this implies that $\operatorname{supp}\left(\gamma_{n}\right)$ is compact for $n \geq m$, and therefore the intersection $\bigcap_{n} \operatorname{supp}\left(\gamma_{n}\right)$ is a non-empty subset of $\bigcap_{n} \operatorname{supp}\left(g_{n}\right)=\{y\}$, so

$$
\bigcap_{n} \operatorname{supp}\left(\gamma_{n}\right)=\{y\} .
$$

It now follows from Lemma 3 that $\left(\gamma_{n}\right)_{n}$ is a characteristic sequence of functions at $y$. In particular, the support of $\gamma_{2}$ is a neighbourhood of $y$. We can therefore choose $j \geq 2$ such that $\operatorname{supp}\left(g_{j}\right)$ is a subset of $\operatorname{supp}\left(\gamma_{2}\right)$, which implies that

$$
\gamma_{1} g_{j}=g_{j}
$$

because $\gamma_{1}$ equals 1 on $\operatorname{supp}\left(\gamma_{2}\right)$ and therefore also on $\operatorname{supp}\left(g_{j}\right)$. This equation implies

$$
\alpha_{1} f_{j}=f_{j}
$$


since $T\left(\gamma_{1}\right)=\alpha_{1} f_{1}$ and $f_{1} f_{j}=f_{j}$. It follows that $\operatorname{supp}\left(f_{j}\right) \subset \operatorname{supp}\left(\alpha_{1}\right) \subset U$. This shows that $\left(f_{n}\right)_{n}$ is a characteristic sequence of functions at $x$. In particular, we have $K=\{x\}$.

(ii) For any $n=1,2, \ldots$ write $f_{n}=T\left(g_{n}\right)$ and $f_{n}^{\prime}=T\left(g_{n}^{\prime}\right)$. Part (i) implies that $\left(f_{n}\right)_{n}$ is a characteristic sequence of functions on $M$ at a point $x \in M$, and that $\left(f_{n}^{\prime}\right)_{n}$ is a characteristic sequence of functions on $M$ at a point $x^{\prime} \in M$. We claim that $x=x^{\prime}$. Indeed, if $x \neq x^{\prime}$, we can choose $m \geq 1$ such that $f_{m} f_{m}^{\prime}=0$. This implies that $g_{m} g_{m}^{\prime}=0$, a contradiction.

Proof of Theorem 1. For any $x \in M$, we choose a characteristic sequence $\left(f_{n}\right)_{n}$ of functions on $M$ at $x$. By Lemma 4 (i) we know that $\left(T^{-1}\left(f_{n}\right)\right)_{n}$ is a characteristic sequence of functions on $N$ at a point of $N$; denote this point by $\tau(x)$. By Lemma 4 (ii), this definition of $\tau(x)$ is independent of the choice of $\left(f_{n}\right)_{n}$; thus we get a map

$$
\tau: M \longrightarrow N \text {. }
$$

We shall now show that $T$ is given by the composition with $\tau$. Take any $x \in M$ and choose a characteristic sequence $\left(f_{n}\right)_{n}$ of functions on $M$ at $x$.

First observe that if $p \in C^{\infty}(N, \mathbb{F})$ is such that $T(p)(x)=0$, then $p(\tau(x))=0$. Indeed, if $p(\tau(x)) \neq 0$, then $p$ has no zeros on an open neighbourhood $V$ of $\tau(x)$ in $N$. We can then choose $n$ so large that $\operatorname{supp}\left(T^{-1}\left(f_{n}\right)\right)$ is a compact subset of $V$, and we can define a smooth function $q \in C^{\infty}(N, \mathbb{F})$ with $\operatorname{supp}(q) \subset V$ by

$$
q(y)=\frac{T^{-1}\left(f_{n}\right)(y)}{p(y)}
$$

for any $y \in V$. Now we have

$$
1=f_{n}(x)=T\left(T^{-1}\left(f_{n}\right)\right)(x)=T(p q)(x)=T(p)(x) T(q)(x),
$$

so $T(p)(x) \neq 0$.

For any $g \in C^{\infty}(N, \mathbb{F})$ we have

$$
T(g-T(g)(x) 1)(x)=T(g)(x)-T(g)(x)=0 .
$$

By the argument above this yields

$$
(g-T(g)(x) 1)(\tau(x))=0,
$$

and therefore

$$
T(g)(x)=g(\tau(x)) .
$$

This shows that $T$ is indeed given by composition with the map $\tau$.

The uniqueness of such a map $\tau$ is clear. Analogously, the isomorphism of algebras $T^{-1}$ is also given by composition with a unique map $N \rightarrow M$, which is the inverse of $\tau$ by uniqueness. Since the smoothness of functions is preserved under the composition with $\tau$ and with its inverse as well, it follows that $\tau$ and its inverse are smooth maps.

Remark 1. The argument above also proves Theorem 2 if we use only functions with compact support. In particular, in the proof of Theorem 1 we can replace the constant function 1 on $N$ with $T^{-1}\left(f_{n}\right)$. 


\section{REFERENCES}

[1] R. Bkouche, Idéaux mous d'un anneau commutatif. Applications aux anneaux de fonctions, C. R. Acad. Sci. Paris 260 (1965), 6496-6498. MR31:1268

[2] N. Dunford and J. T. Schwartz, Linear operators. Part I, Wiley Classics Library, John Wiley \& Sons Inc., New York, 1988, General theory, With the assistance of William G. Bade and Robert G. Bartle, Reprint of the 1958 original, A Wiley-Interscience Publication. MR.90g:47001a

[3] I. Gelfand and A. Kolmogoroff, On rings of continuous functions on topological spaces, C. R. (Doklady) Acad. Sci. URSS (N. S.) 22 (1939), 11-15.

[4] J. Mrčun, On spectral representation of coalgebras and Hopf algebroids, Preprint arXiv: math.QA/0208199 (2002).

[5] J. Nagata, On lattices of functions on topological spaces and of functions on uniform spaces, Osaka Math. J. 1 (1949), 166-181. MR11:185c

[6] G. Shilov, Ideals and subrings of the ring of continuous functions, C. R. (Doklady) Acad. Sci. URSS (N. S.) 22 (1939), 7-10.

Department of Mathematics, University of Ljubljana, Jadranska 19, 1000 Ljubljana, SLOVENIA

E-mail address: janez.mrcun@fmf.uni-lj.si 